\title{
Ultrashort Optical Solitons in Nonlinear Media with Arbitrary Dispersion
}

\author{
Shalva Amiranashvili and Uwe Bandelow \\ Weierstrass Institute for Applied Analysis and Stochastics (WIAS), Mohrenstr. 39, 10117 Berlin, Germany \\ Nail Akhmediev \\ Optical Sciences Group, Research School of Physics and Engineering, Institute of Advanced Studies, \\ The Australian National University, Canberra ACT 0200, Australia
}

\begin{abstract}
We consider propagation of ultrashort optical pulses in nonlinear fibers and suggest a new theoretical framework for description of pulse dynamics and exact characterization of solitary solutions. Our approach deals with a proper complex generalization of the nonlinear Maxwell equations and completely avoids the use of the slowly varying envelope approximation. The only essential restriction is that fiber dispersion does not favor both the so-called Cherenkov radiation, as well as the resonant generation of the third harmonics, as these effects destroy ultrashort solitons. Assuming that it is not the case, we derive a continuous family of solitary solutions connecting fundamental solitons to nearly single-cycle ultrashort ones for arbitrary anomalous dispersion and cubic nonlinearity.
\end{abstract}

To find a real-valued solution of, e.g., a linear wave equation, one often looks for a complex-valued solution first. This useful technique can be generalized to nonlinear models. For instance, consider a complex-valued solution $\mathcal{E}(z, t)$ of the equation

$$
\partial_{z}^{2} \mathcal{E}-\frac{1}{c^{2}} \partial_{t}^{2}\left(\hat{\epsilon} \mathcal{E}+\frac{3}{4} \chi^{(3)}|\mathcal{E}|^{2} \mathcal{E}+\frac{1}{4} \chi^{(3)} \mathcal{E}^{3}\right)=0
$$

where a nonlocal operator $\hat{\epsilon}$ represents arbitrary dispersion, $(\hat{\epsilon} \mathcal{E})_{\omega}=\epsilon(\omega) \mathcal{E}_{\omega}$, and for simplicity we additionally assume that $\operatorname{Im}[\epsilon(\omega)] \approx 0$ and $\chi^{(3)} \approx$ const for the frequency range of interest. One can directly check that $\mathcal{E}(z, t)$ yields a realvalued solution $E(z, t)=\operatorname{Re}[\mathcal{E}(z, t)]$ of the standard nonlinear wave equation

$$
\partial_{z}^{2} E-\frac{1}{c^{2}} \partial_{t}^{2}\left(\hat{\epsilon} E+\chi^{(3)} E^{3}\right)=0
$$

e.g., for a single-mode nonlinear fiber with the effective (i.e., including both bulk medium dispersion and geometry effects) dispersion function $\epsilon(\omega)$.

Notably, the complex field in Eq. (1) is introduced without the use of the slowly varying envelope approximation (SVEA). The complex Eq. (1) may be considered as an unusual way to solve the real Eq. (2). The main advantage of the approach is that Eq. (1) explicitly distinguishes between two different 4-wave mixing processes: self-phase modulation (SPM) and third harmonics generation (THG). Moreover, for a favorable dispersion function one can neglect THG by omitting the $\mathcal{E}^{3}$ term in Eq. (1). It is then convenient to relate $\mathcal{E}(z, t)$ to the analytic signal for the electric field by taking a suitable initial condition. By construction we set

$$
\left.\mathcal{E}(z, t)\right|_{z=0}=\left.2 \sum_{\omega>0} e^{-i \omega t} E_{\omega}(z)\right|_{z=0}
$$
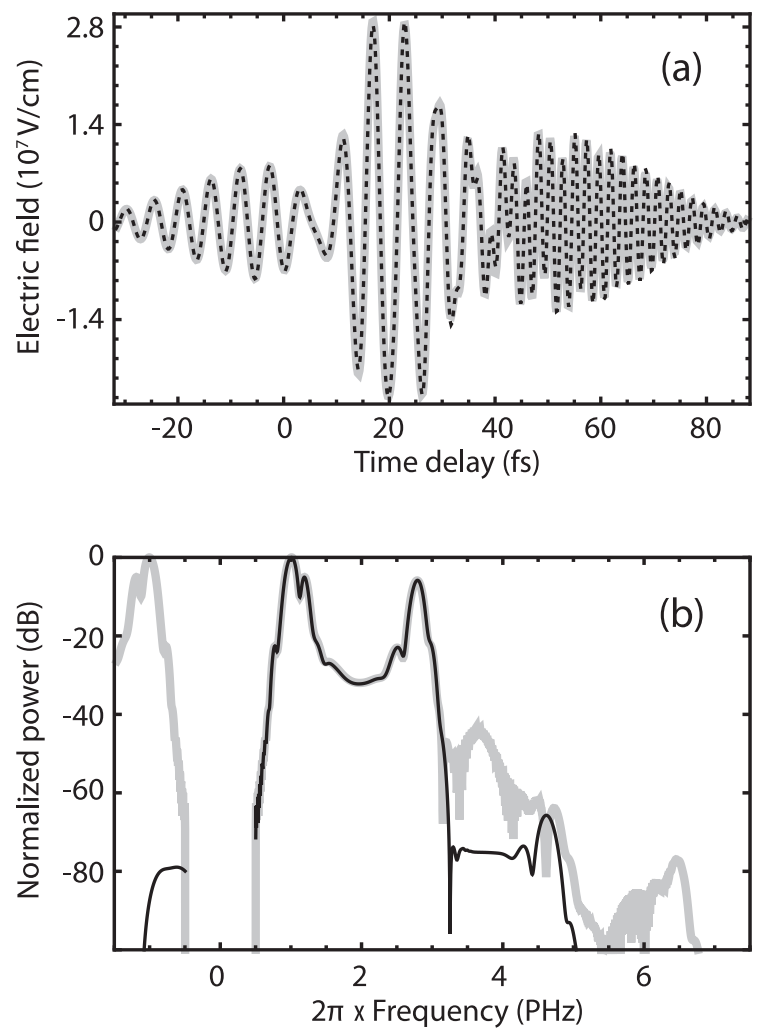

Fig. 1. An exemplary solution of Eq. (1) without $\mathcal{E}^{3}$ term (black lines) compared to the full solution of the wave equation (2) (gray lines). An initially sech-shaped ultrashort pulse is shown at $z=3000 \mu \mathrm{m}$, for further details see [1]. (a) good coincidence in the time domain; (b) good coincidence for positive frequencies in the spectral domain. The negative-frequency part of $\mathcal{E}(z, t)$ remains small indicating that the complex field is a very close approximation to the analytic signal for $E(z, t)$.

such that $\left.\mathcal{E}_{\omega}(z)\right|_{z=0}$ vanishes for $\omega<0$. The summation in Eq. (3) is performed over all discrete positive frequencies corresponding to a large period of the pulse sequence in the time domain.

One can demonstrate that in the absence of the resonant THG the negative-frequency part of $\mathcal{E}(z, t)$ remains small in the course of pulse propagation [1]. Therefore to a good approximation $\mathcal{E}(z, t)$ is determined exclusively by SPM and represents the analytic signal ("fast envelope") for the real- 

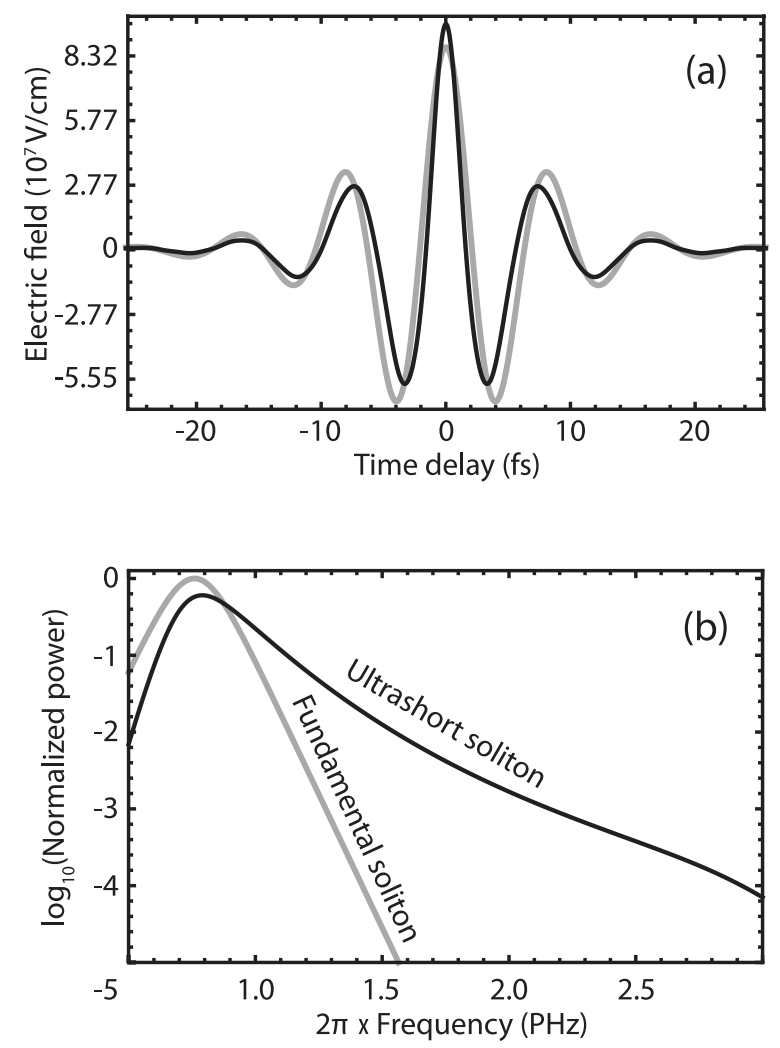

Fig. 2. (a) A single-cycle soliton at $\lambda=2.5 \mu \mathrm{m}$ calculated for bulk fused silica dispersion (for further details see [1]) is shown by the black curve. For comparison, the corresponding fundamental NLSE soliton solution is shown by the grey curve. (b) Spectral densities of the same solutions, the difference is now clearly observable.

valued electric field. This is illustrated in Fig. 1 showing for $z=3000 \mu \mathrm{m}$ an initially $14 \mathrm{fs}$ (FWHM) sech-shaped pulse at $\lambda=1.6 \mu \mathrm{m}$ propagating along bulk fused silica. The initial pulse amplitude is twice that of the fundamental soliton, the initial peak power $\approx 2 \times 10^{12} \mathrm{~W} / \mathrm{cm}^{2}$.

Equation (1) can be considered as a kind of "bidirectional nonlinear Schrödinger equation" (bidirectional NLSE) which is free of SVEA. We used Eq. (1) to study ultrashort optical solitons in a focusing nonlinear media with cubic nonlinearity and an arbitrary anomalous $\epsilon(\omega)$ (see [1]). The well known fundamental soliton solutions are described by the standard NLSE which is derived under SVEA [2]-[4]. These solitons are organized in a continuous family of solutions [5]; they are parametrized, among other things, by the pulse duration. As the pulse duration decreases, the soliton is transformed into a few-cycle pulse such that both the SVEA and the polynomial representation of the dispersion function become invalid [6], [7]. At this point our Eq. (1) comes into play.

The solitary solutions were found using the spectral renormalisation method originally developed for the envelope solitons [8]. To this end we first substitute a standard soliton ansatz into Eq. (1). The resulting equation is reformulated in an iterative manner $\mathcal{E}=\hat{\mathcal{N}}[\mathcal{E}]$ where $\hat{\mathcal{N}}$ is a suitable nonlinear operator. The iterations start with the fundamental soliton and are performed in two steps

$$
\mathcal{E}_{n} \rightarrow \mathcal{E}_{n+1 / 2}=\hat{\mathcal{N}}\left[\mathcal{E}_{n}\right] \rightarrow \mathcal{E}_{n+1}=s_{n} \mathcal{E}_{n+1 / 2},
$$

where the rescaling factor $s_{n}$ in the second step is chosen in such a way that

$$
\left\langle\mathcal{E}_{n+1} \mid \mathcal{E}_{n+1}\right\rangle=\left\langle\mathcal{E}_{n} \mid \mathcal{E}_{n}\right\rangle,
$$

for a suitably defined scalar product, e.g., keeping the photon number constant. The successive iterations $\mathcal{E}_{n}$ are then forced to belong to a kind of "unit sphere", this greatly improves (but not ensures!) convergence of the method.

An exemplary solution for the bulk fused silica dispersion and carrier frequency at $\lambda=2.5 \mu \mathrm{m}$ is shown in Fig. 2 . The difference between the exact soliton and the simplest fundamental one is well pronounced when plotted in the frequency domain. In conclusion our approach allows to trace solitons up to a nearly single-cycle duration. Too short solitons are finally destroyed either by Cherenkov radiation [9], [10], or by cusp formation [11], [12].

\section{ACKNOWLEDGMENT}

The work of Sh. Amiranashvili has been supported by the Deutsche Forschungsgemeinschaft (DFG) within the collaborative research center MATHEON under Grant D14. N.A. acknowledges the support of the Australian Research Council (Discovery Project DP110102068) and the Volkswagen Stiftung. He is also a recipient of the Alexander von Humboldt Award.

\section{REFERENCES}

[1] S. Amiranashvili, U. Bandelow, and N. Akhmediev, "Few-cycle optical solitary waves in nonlinear dispersive media," Phys. Rev. A, vol. 87, no. 1, p. 013805, Januar 2013.

[2] A. Hasegawa, Optical Solitons in Fibers. Springer, 1980.

[3] N. Akhmediev and A. Ankiewicz, Solitons: Nonlinear Pulses and Beams. Chapman and Hall, 1997.

[4] G. P. Agrawal, Nonlinear Fiber Optics, 4th ed. New York: Academic, 2007.

[5] V. E. Zakharov and A. B. Shabat, "Exact theory of two-dimensional self-focusing and one-dimensional self-modulation of waves in nonlinear media," Sov. Phys. JETP, vol. 34, no. 1, pp. 62-69, 1972.

[6] K. E. Oughstun and H. Xiao, "Failure of the quasimonochromatic approximation for ultrashort pulse propagation in a dispersive, attenuative medium," Phys. Rev. Lett., vol. 78, no. 4, pp. 642-645, 1997.

[7] S. Amiranashvili, U. Bandelow, and A. Mielke, "Padé approximant for refractive index and nonlocal envelope equations," Opt. Comm., vol. 283, no. 3, pp. 480-485, 2010.

[8] M. J. Ablowitz and T. P. Horikis, "Solitons and spectral renormalization methods in nonlinear optics," Eur. Phys. J. Special Topics, vol. 173, pp. 147-166, 2009.

[9] N. Akhmediev and M. Karlsson, "Cherenkov radiation emitted by solitons in optical fibers," Phys. Rev. A, vol. 51, no. 3, pp. 2602-2607, 1995.

[10] D. V. Skryabin and A. V. Gorbach, "Colloquium: Looking at a soliton through the prism of optical supercontinuum," Rev. Mod. Phys., vol. 82, no. 2, pp. 1287-1299, 2010.

[11] S. Amiranashvili, A. G. Vladimirov, and U. Bandelow, "Solitary-wave solutions for few-cycle optical pulses," Phys. Rev. A, vol. 77, no. 6, p. 063821,2008

[12] S. Amiranashvili, U. Bandelow, and N. Akhmediev, "Dispersion of nonlinear group velocity determines shortest envelope solitons," Phys. Rev. A, vol. 84, no. 4, p. 043834, 2011. 